\title{
De Novo Regeneration of Scrophularia yoshimurae YAMAZAKI (Scrophulariaceae) and Quantitative Analysis of Harpagoside, an Iridoid Glucoside, Formed in Aerial and Underground Parts of In Vitro Propagated and Wild Plants by HPLC
}

\author{
Abhay P. SAgare, ${ }^{a}$ Chao-Lin Kuo,${ }^{b, c}$ Fu-Shin ChueH, ${ }^{a}$ and Hsin-Sheng TsaY ${ }^{*, a}$ \\ Department of Agronomy, Taiwan Agricultural Research Institute, ${ }^{a}$ Wufeng, Taichung 41301, Taiwan, Chung Hwa College \\ of Medical Technology, ${ }^{b}$ Jen-Te Hsiang, Tainan Hsien, Taiwan, and Institute of Chinese Pharmaceutical Science, China \\ Medical College, ${ }^{c}$ Taichung 40421, Taiwan. Received June 4, 2001; accepted August 28, 2001
}

\begin{abstract}
A protocol for de novo regeneration and rapid micropropagation of Scrophularia yoshimurae (Scrophulariaceae) has been developed. Multiple shoot development was achieved by culturing the shoot-tip, leaf-base, stemnode and stem-internode explants on Murashige and Skoog (MS) medium supplemented with $4.44 \mu \mathrm{M} N^{6}$-benzyladenine (BA) and 1.07 $\mu_{\mathrm{M}} \alpha$-naphthaleneacetic acid (NAA). Stem-node and shoot-tip explants showed the highest response $(100 \%)$ followed by stem-internode $(74.4 \%)$ and leaf-base $(7.7 \%)$ explants. The shoots were multiplied by subculturing on the same medium used for shoot induction. Shoots were rooted on growth regulator-free MS basal medium and the plantlets were transplanted to soil and acclimatized in the growth chamber. The content of harpagoside, a quantitatively predominant iridoid glycoside, in different plant material was determined by high performance liquid chromatography (HPLC). The analysis revealed that the content of harpagoside in the aerial and underground parts of $S$. yoshimurae was significantly higher than the marketed crude drug (underground parts of Scrophularia ningpoensis).
\end{abstract}

Key words germplasm conservation; harpagoside; Scrophularia yoshimurae; Scrophularia ningpoensis; Scrophulariaceae; micropropagation

The family Scrophulariaceae comprises of some important medicinal plants such as Bacopa monnieri, ${ }^{1)}$ Digitalis spp., ${ }^{2)}$ Rehmannia glutinosa ${ }^{3)}$ and Scrophularia spp. Species of the genus Scrophularia have been used as crude drug in traditional medicine. ${ }^{4-7)}$

Scrophularia ningpoensis HemsLey, commonly known as "Xuanshen" in Chinese, is an important medicinal plant. The roots of $S$. ningpoensis have been used in the traditional Chinese medicine in the treatment of inflammation, laryngitis, tonsillitis, abscesses of carbuncles ${ }^{8)}$ and constipation. ${ }^{9)}$ It can lower blood pressure ${ }^{4,10,11)}$ and blood sugar levels and also has antibacterial and antioxidant effects. ${ }^{10,11)}$ Small doses of S. ningpoensis are cardiotonic ${ }^{8)}$ and it is commonly used in combination with other herbs as nutrient and health strengthening agent. ${ }^{10)}$ The root contains iridoid glycosides, which are active principles, and small amounts of essential oils, alkaloids, flavanoids, and $p$-methoxycinnamic acid. ${ }^{10)}$

Scrophularia yoshimurae YAMAZAKI, a herbaceous perennial plant $40-60 \mathrm{~cm}$ tall, is indigenous to Taiwan. $S$. yoshimurae is used as "Xuanshen", a substitute for S. ningpoensis, in traditional Chinese medicine in Taiwan. ${ }^{12)}$ Populations of $S$. yoshimurae are distributed in the central mountain range of Taiwan and they are adapted to a narrow set of environmental conditions, found only at middle elevations (altitude about $1000-1300 \mathrm{~m}$ ). ${ }^{13)}$ It is very difficult to locate plants of S. yoshimurae in wild. In Taiwan, the processed roots of Scrophularia ningpoensis are imported from mainland China and used as crude drug as $S$. yoshimurae is not cultivated on commercial scale and the roots collected from plants growing naturally in the mountains of Taiwan are insufficient to meet the local demand. Development of a rapid in vitro propagation system for $S$. yoshimurae would help in commercial cultivation and conserving the germplasm of this medicinally important species.
In vitro culture techniques have been used successfully for propagation of many medicinally important plant species either by shoot morphogenesis ${ }^{2,3,14-17)}$ or somatic embryogenesis. ${ }^{18-20)}$ Plants propagated by in vitro techniques showed less variation in the content of secondary metabolites than its cultivated/wild counterpart. ${ }^{16)}$ Reports on in vitro propagation of medicinally important plants of Scrophulariaceae are available. ${ }^{1-3,21,22)}$ However, to our knowledge, in vitro regeneration in Scrophularia spp. has not been reported. The aims of the present study were (i) to standardize an efficient protocol for in vitro propagation of $S$. yoshimurae, and (ii) to analyse the content of harpagoside (Fig. 1), a quantitatively predominant iridoid glycoside, in the commercially available crude drug (Scrophulariae radix, processed roots of S. ningpoensis) and aerial and underground parts of wild and in vitro propagated plants of $S$. yoshimurae.

\section{MATERIALS AND METHODS}

Plant Material Flowering and non-flowering plants of Scrophularia yoshimurae YAMAZAKI were collected from Chitou, Nantou County in central Taiwan (altitude about $1300 \mathrm{~m}$ ) in September 1995 and December 2000. A voucher specimen of the flowering plant (No. CMC SY 0111) has been deposited at the China Medical College herbarium, Taichung, Taiwan. Plants collected in September 1995 were replanted in $18-\mathrm{cm}$ diameter pots in potting mixture soil:

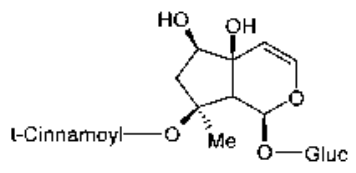

Fig. 1. The Chemical Structure of Harpagoside 
peat moss: vermiculite $(1: 1: 1 \mathrm{v} / \mathrm{v} / \mathrm{v})$ and the containers were kept in the growth chamber (Hotech Instruments Corp., Model 624 HD, Taipei, Taiwan) under a light intensity of $100 \mu \mathrm{mol} \cdot \mathrm{m}^{-2} \cdot \mathrm{s}^{-1}, 16-\mathrm{h}$ photoperiod per day and $20 / 16^{\circ} \mathrm{C}$ day/night temperature. The plants were watered once a week with tap water. Three samples of the crude drug "Scrophulariae Radix" (underground parts of Scrophularia ningpoensis Hemsley) were procured from the drug market in Taichung, Taiwan.

Tissue Culture New shoots $25-30 \mathrm{~cm}$ in length from the replanted plants were used as a source of explants for in vitro culture. Shoot-tip, leaf-base, stem-node and stem-internode explants ( $5 \mathrm{~mm}$ long) were used for the induction of shoots. All the explants were surface disinfected in $70 \%$ ethanol for $30 \mathrm{~s}$, followed by treatment with $0.5 \%$ sodium hypochlorite (with one drop of Tween $20^{\circledR}$ per $100 \mathrm{ml}$ ) under ultrasonic vibration for $5 \mathrm{~min}$ and rinsed five times with sterile distilled water. All explants were cultured in $22 \times 120-\mathrm{mm}$ glass test tubes, each containing $10-\mathrm{ml}$ medium. The medium consisted of Murashige and Skoog (MS) $\left.{ }^{23}\right)$ basal medium supplemented with 3\% sucrose, $0.9 \%$ Difco Bacto ${ }^{\mathrm{TM}}$ Agar (Difco Laboratories, Detroit, Michigan, U.S.A.), $N^{6}$-benzyladenine (BA) at $4.44 \mu \mathrm{M}$ and $\alpha$-naphthaleneacetic acid (NAA) at $1.07 \mu \mathrm{M}$ (Table 1). The culture vessels were capped with two layers of aluminum foil before autoclaving, and sealed with three layers of Parafilm $M$ after culture. Twenty explants were evaluated for induction of shoots. The percentage of explants producing shoots and the average number of shoots produced per explant were recorded. Shoots produced from shoot-tip, axillary buds of the nodal explants, leaf-base and stem-internode explants were multiplied by culturing in 250-ml Erlenmeyer flasks containing 100-ml shoot induction medium for one month. Eight shoots were cultured per flask. Forty explants were evaluated for multiplication of shoots. Flasks were plugged with cotton. After one month of culture in shoot multiplication medium, clumps of multiple shoots were subcultured and incubated for 3 weeks on growth regulator-free MS basal medium with 3\% sucrose, $0.9 \%$ Difco Bacto Agar in 250-ml Erlenmeyer flasks for elongation. For rooting, $1.5-2.0-\mathrm{cm}$-long shoots were cultured on growth regulator-free $\mathrm{MS}$ basal medium with $3 \%$ sucrose, $0.9 \%$ Difco Bacto Agar in 500-ml Erlenmeyer flasks and incubated for 3 weeks. Eight shoots were cultured per flask. The pH of all media was adjusted to $5.7 \pm 0.1$ with $1 \mathrm{~N} \mathrm{NaOH}$ before autoclaving at $121^{\circ} \mathrm{C}, 105 \mathrm{kPa}$ for $15 \mathrm{~min}$. All the cultures were incubated at $25 \pm 1{ }^{\circ} \mathrm{C}$ under cool white fluorescent light at $38 \mu \mathrm{mol} \cdot \mathrm{m}^{-2} \cdot \mathrm{s}^{-1}$ (Philips, Holland) with a 16 -h photoperiod per day. Ten plantlets with well-developed roots were removed from flasks and washed thoroughly under tap water for $2-3$ min to remove traces of agar-gelled medium sticking to them. Plants were then dipped in $0.1 \%(\mathrm{w} / \mathrm{v})$ Benlate $^{\circledR}$ (Du Pont, De Nemours \& Co. Inc., Taoyuan, Taiwan) for a minute and transferred to $9-\mathrm{cm}$ diameter plastic containers with a mixture of autoclaved soil : peat moss : vermiculite (1 : $1: 1 \mathrm{v} / \mathrm{v} / \mathrm{v})$ and the containers were kept in the growth chamber (Hotech Instruments Corp., Model 624 HD, Taipei, Taiwan) under a light intensity of $100 \mu \mathrm{mol} \cdot \mathrm{m}^{-2} \cdot \mathrm{s}^{-1}, 16$-h photoperiod per day and $20 / 16^{\circ} \mathrm{C}$ day/night temperature for 3 weeks. The plants were initially covered with polypropylene bags for one week to maintain humidity and prevent wilting. After 3 weeks of incubation in the growth chamber, the plants were transplanted to $18-\mathrm{cm}$ diameter pots containing the same potting mixture and grown in the growth chamber for one year under the conditions described earlier. The plants were irrigated once in a week with tap water. The plants were fertilized once in a month with $\mathrm{HYPONeX}^{\circledR}$ No. 2 (Hyponex Corporation, Marysville, OH 43041, U.S.A.).

Standard Compound Authentic, HPLC grade harpagoside compound for calibration was obtained from Extrasynthese (Genay, France).

Extraction and Quantitative Analysis of Harpagoside The aerial and underground parts of wild flowering and nonflowering plants (collected in December 2000) and tissue cultured plants of $S$. yoshimurae were carefully cleaned, then dried in a hot air oven at about $40{ }^{\circ} \mathrm{C}$ for three days. The dried aerial and underground parts $(200 \mathrm{mg})$ of different plant material (Table 2) were finely ground with mortar and pestle and extracted 5 times at room temperature with methanol $(10 \mathrm{ml})$. The extract was filtered through an Advantec No. 1 filter paper (Toyo Roshi Kaisha Ltd., Japan) and the methanol was evaporated in vacuo to dryness. The residue of the combined extracts was re-dissolved in $5 \mathrm{ml}$ methanol, filtered through a membrane filter $\left(0.2 \mu \mathrm{m}\right.$ pore size, Nalgene ${ }^{\circledR}$, New York, U.S.A.) and $10 \mu \mathrm{l}$ of the solution was subjected to HPLC. Analysis was performed on a Waters high-performance liquid chromatograph (Waters ${ }^{\mathrm{TM}}$, Milford, Massachusetts, U.S.A.), which was connected to a Waters Symmetry ${ }^{\circledR}$ $\mathrm{C}_{18} 5 \mu \mathrm{m}, 3.9 \times 150 \mathrm{~mm}$ HPLC column (Waters Corporation, Milford, U.S.A.) fitted with a Sentry ${ }^{\mathrm{TM}}$ guard precolumn, Symmetry ${ }^{\circledR} \mathrm{C}_{18} 5 \mu \mathrm{m}, 3.9 \times 20 \mathrm{~mm}$ (Waters). The HPLC system consisted of a Waters ${ }^{\mathrm{TM}} 600$ controller, Waters $^{\mathrm{TM}} 717$ plus autosampler, a 996 photo diode array variable wave length detector and Millennium ${ }^{32}$ software. The mobile phase (methanol: water $=50: 50$ ) was pumped at a flow rate of $0.8 \mathrm{ml}$ per min. Ultraviolet (UV) detection was made at $278.9 \mathrm{~nm}$. The cycle time of analysis was about $30 \mathrm{~min}$. Harpagoside $(10 \mathrm{mg})$ was dissolved in methanol $(1 \mathrm{mg} / \mathrm{ml})$, diluted stepwise $(1,0.5,0.25,0.125$, and $0.0625 \mathrm{mg} / \mathrm{ml})$, and $10 \mu \mathrm{l}$ portions were injected into the HPLC. The calibration plot was obtained by measuring their respective peak areas.

Statistical Analysis All treatments were repeated three times. For statistical analysis, shoot number and length were recorded. Least significant difference (LSD) test or standard deviation was used for statistical analysis.

\section{RESULTS AND DISCUSSION}

Tissue Culture Plants which were used as a source of explants for in vitro studies were grown under controlled conditions and explants were taken from actively growing shoots as described in earlier studies with Limonium wrightii $^{24)}$ and Adenophora triphylla. ${ }^{25)}$ To induce multiple shoots, stem-internode, stem-node, shoot-tip and leaf-base explants of $S$. yoshimurae were cultured on MS basal medium with $4.44 \mu \mathrm{M}$ BA and $1.07 \mu \mathrm{M}$ NAA (Table 1). A medium supplemented with BA and NAA has been used earlier for in vitro induction of shoots in some medicinal plants. ${ }^{15,24,25)}$ In $S$. yoshimurae, multiple shoots were induced not only from pre-existing meristems of the shoot-tip and stem-node explants but also developed from the cut end of the stem-internode and leaf-base explants without an intervening callus phase (Table 1, Fig. 2). Figure 2A shows ad- 
ventitious shoots (arrows) induced directly from the cut end of stem-internode (si) explant after one month of culture. Adventitious shoots (s) and shoot buds (arrow) were also developed directly at the cut end of leaf-base (lb) explants

Table 1. Effect of Explant Type on Shoot Regeneration in Scrophularia yoshimurae YAMAZAKI after $30 \mathrm{~d}$ of Culture on MS Medium Supplemented with $4.44 \mu \mathrm{m}$ BA and $1.07 \mu \mathrm{M}$ NAA

\begin{tabular}{lcc}
\hline \hline \multicolumn{1}{c}{ Explant } & $\begin{array}{c}\text { \% Explants producing } \\
\text { shoot buds }\end{array}$ & $\begin{array}{c}\text { Average no. shoot } \\
\text { buds per explant }\end{array}$ \\
\hline Stem-internode & 71.4 & $2.80 \mathrm{a}$ \\
Stem-node & 100.0 & $3.43 \mathrm{a}$ \\
Shoot-tip & 100.0 & $2.35 \mathrm{a}$ \\
Leaf-base & 7.7 & $0.12 \mathrm{~b}$ \\
\hline
\end{tabular}

Means within a column followed by a same letter are not significantly different from each other at $5 \%$ level by LSD (least significant difference) test. along with adventitious roots (r) (Fig. 2B). However, the percentage of leaf-base explants showing induction of adventitious shoots was lower than stem-internode explants (Table 1). Shoots obtained after one month of culture of different explants were separated and transferred onto the same shoot induction medium for proliferation. After one month of culture on shoot multiplication medium, the average number of shoots produced per shoot cultured was $10.3 \pm 1.0$. Since the average length of shoots was only $8.6 \pm 0.2 \mathrm{~mm}$, multiple shoots were transferred to MS basal medium without growth regulators for elongation. Fig. $2 \mathrm{C}$ shows elongated shoots after three weeks culture on growth regulator-free medium. Roots in micropropagated shoots were induced by culturing elongated shoots again on growth regulator-free MS medium. Root development occurred from the base of the shoots after three weeks of culture without callus formation at the cut end
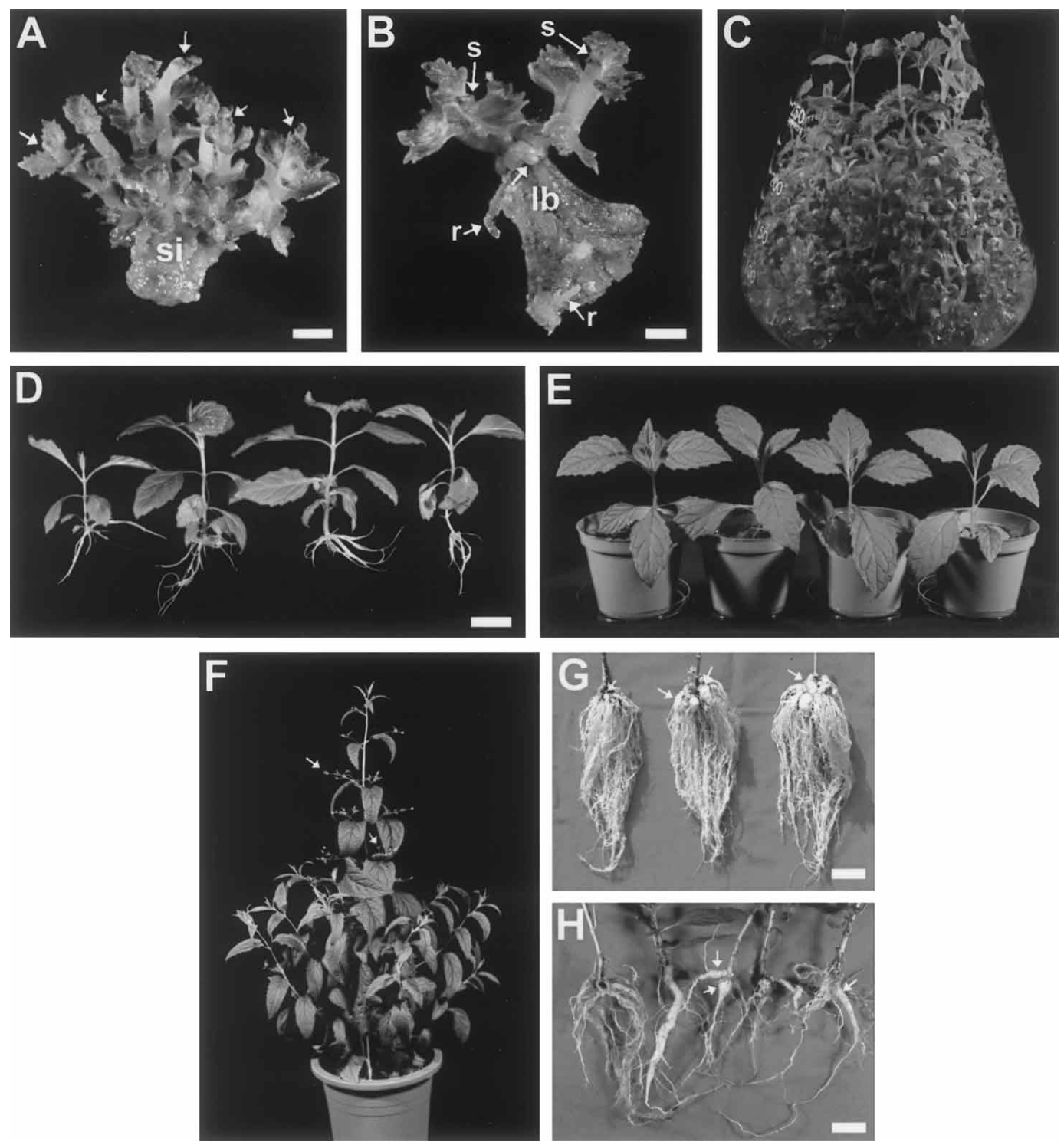

Fig. 2. De Novo Regeneration and Micropropagation of Scrophularia yoshimurae YAMAZAKI

(A) Induction of shoots (arrows) from stem-internode (si) explant on MS basal medium supplemented with 4.44 $\mu \mathrm{M}$ BA and 1.07 $\mu \mathrm{M}$ NAA. Bar, 2.8 mm. (B) Induction of shoots (s), shoot buds (arrow) and roots (r) from leaf-base (lb) explant on MS basal medium supplemented with 4.44 $\mu \mathrm{M}$ BA and 1.07 $\mu \mathrm{M}$ NAA. Bar, $3.4 \mathrm{~mm}$. (C) Elongation of shoots in MS basal medium without growth regulators. Shoots induced on different explants were multiplied in MS medium supplemented with $4.44 \mu \mathrm{M}$ BA and 1.07 $\mu \mathrm{M}$ NAA before culture on MS basal medium without growth regulators. (D) Rooted shoots after three weeks of culture on growth regulator-free MS basal medium. Bar, $2.75 \mathrm{~cm}$. (E) In vitro regenerated plantlets three weeks after transfer to soil : peat moss : vermiculite $(1: 1: 1 \mathrm{v} / \mathrm{v} / \mathrm{v})$ in 9 -cm diameter plastic pot. (F) In vitro regenerated plant after transfer to 18 -cm diameter pot and 1 year of growth in the growth chamber. Note flowering (arrows) in the plant. (G) Well-developed fleshy roots (arrows) in in vitro propagated plant after 1 year of growth in the growth chamber. Bar, $4.86 \mathrm{~cm}$. (H) Fleshy roots (arrows) in flowering plants of $S$. yoshimurae collected from the natural habitat. Bar, $2.38 \mathrm{~cm}$. 
Table 2. Content of Harpagoside in Underground and Aerial Parts of Wild and In Vitro Propagated Plants of Scrophularia yoshimurae YAMAZAKI and in Underground Parts of Scrophularia ningpoensis HEMSLEY

\begin{tabular}{lcc}
\hline \hline & \multicolumn{2}{c}{ Harpagoside $(\mathrm{mg} / \mathrm{g}$ dry weight $)$} \\
\cline { 2 - 3 } & Underground parts & Aerial parts \\
\hline S. ningpoensis & $0.49 \pm 0.03$ & $\left.-{ }^{a}\right)$ \\
(Crude drug) & & \\
S. yoshimurae & & $0.75 \pm 0.06$ \\
$\mathrm{~A}^{b)}$ & $3.44 \pm 0.04$ & $4.12 \pm 0.28$ \\
$\mathrm{~B}^{b)}$ & $4.92 \pm 0.16$ & $4.07 \pm 0.20$ \\
$\mathrm{C}^{b)}$ & $5.48 \pm 0.35$ &
\end{tabular}

a) Not analysed. b) A, in vitro rooted plants with flowers after one year of transplantation in the growth chamber; B, wild non-flowering plant; $\mathrm{C}$, wild flowering plant, $\pm=$ standard deviation.

(Fig. 2D), similar to earlier observations. ${ }^{26}$ The percentage rooting of shoots was $100 \%$. The shoots with well-developed root system (Fig. 2D) were transplanted to a potting mixture of soil:peat moss: vermiculite $(1: 1: 1 \mathrm{v} / \mathrm{v} / \mathrm{v})$. The in vitro plantlets became established in potting mixture in three weeks (Fig. 2E) with $100 \%$ survival rate when transferred to the growth chamber under high humidity conditions. Figure $2 \mathrm{~F}$ shows plant with flowering (arrows) after one year of incubation in the growth chamber. All plants grown in the growth chamber showed well-developed fleshy roots (arrows) (Fig. 2G). Figure $2 \mathrm{H}$ shows roots of flowering plants of $S$. yoshimurae collected from wild. Although the roots of wild plants showed development of fleshy roots (arrows), their average length $(10.88 \pm 2.33 \mathrm{~cm})$ was almost three times shorter than the one-year-old in vitro propagated plants $(30.77 \pm$ $1.33 \mathrm{~cm}$ ). The average weight of underground parts in tissue cultured plants after one year of growth was $14.9 \pm 5.8 \mathrm{~g}$ while in wild non-flowering and flowering plants it was only $0.23 \pm 0.01 \mathrm{~g}$ and $0.51 \pm 0.23 \mathrm{~g}$ respectively. Wild plants of $S$. yoshimurae grow in hilly region with shallow sandy soil. By contrast, in vitro propagated plants were grown in soil under controlled environmental conditions and fertilized regularly. This may explain the observed differences in the length and weight of roots of wild and in vitro propagated plants of $S$. yoshimurae.

Quantitative Analysis Iridoid glycosides are widespread in the genus Scrophularia. ${ }^{27-29)}$ Among the iridoid glycosides, harpagoside is quantitatively predominant. For quantitative analysis, peak areas were used to calculate the amount of harpagoside present in different plant material as compared to the standard. The calibration plot was linear. The identification was made on the basis of its ultraviolet absorption spectra and retention time. Table 2 shows the contents of harpagoside in different plant material as determined by HPLC analysis. The concentration of harpagoside varied in different plant material. In $S$. yoshimurae the harpagoside content found in aerial parts varied from 0.75 to $4.12 \mathrm{mg} / \mathrm{g}$ dry weight, while in underground parts it varied from 3.44 to $5.48 \mathrm{mg} / \mathrm{g}$ dry weight (Table 2). Isolation of harpagoside (around $0.1 \mathrm{mg} / \mathrm{g}$ dry weight) from the dried roots of $S$. ningpoensis has been reported earlier. ${ }^{28,29)}$ Due to unavailability of intact $S$. ningpoensis plants in Taiwan, we could not analyse the content of harpagoside in its aerial and underground parts. The amount of harpagoside $(0.49 \mathrm{mg}$ per gram dry weight) observed in the underground parts of $S$. ning- poensis (marketed crude drug) was significantly lower than the underground parts of $S$. yoshimurae (Table 2).

\section{CONCLUSIONS}

We have been successful in maintaining shoot cultures of S. yoshimurae for over 2 years without any marked decrease in the rate of shoot multiplication (data not shown). The present study shows that the aerial and underground parts of $S$. yoshimurae contain fairly high amount of harpagoside. Since harpagoside and other iridoid glucosides of Scrophularia spp. have shown anti-inflammatory, ${ }^{30,31)}$ antihepatotoxic and immunostimulant $^{32)}$ properties, in vitro propagated plants of $S$. yoshimurae, which contain fairly high amount of harpagoside, could be used for pharmacological studies. These findings may also be useful in rapid micropropagation, commercial cultivation and germplasm conservation of $S$. yoshimurae. Micropropagated plants of $S$. yoshimurae could be reintroduced in its natural habitat.

Acknowledgements This research was supported by a grant from the National Science Council (NSC 89-2317B010-002), Taiwan. This paper is Taiwan Agricultural Research Institute Contribution No. 2062.

\section{REFERENCES}

1) Tiwari V., Deo Singh B., Nath Tiwari K., Plant Cell Rep., 17, 538543 (1998).

2) Erdei I., Kiss Z., Maliga P., Plant Cell Rep., 1, 34-35 (1981).

3) Shoyama Y., Nagano M., Nishioka I., Planta Med., 48, 124-125 (1983).

4) Kajimoto T., Hidaka M., Shoyama K., Nohara T., Phytochemistry, 28, 2701-2704 (1989)

5) Fernández M. A., García M. D., Sáenz M. T., J. Ethnopharmacol., 53, $11-14$ (1996).

6) Miyase T., Mimatsu A., J. Nat. Prod., 62, 1079-1084 (1999).

7) Giner R. M., Villalba M. L., Recio M. C., Máñez S., Cerdá-Nicolás M., Ríos J. L., Eur. J. Pharmacol., 389, 243-252 (2000).

8) Reid D. P., "Chinese Herbal Medicine," ed. by Amsel A., McClellan M., Maitland D., Shambhala Publications, Inc., Boston, Massachusetts, 1996, p. 98.

9) Yen K. Y., "The Illustrated Chinese Materia Medica Crude and Prepared," SMC Publishing Inc., Taipei, Taiwan, 1992, p. 64.

10) Huang K. C., "The Pharmacology of Chinese Herbs," CRC Press, Boca Raton, FL, 1993, pp. 281-282.

11) Anon., "Chung-Hua-Ben-Tsao," Vol. 7, Shanghai Science Technology Press, Shanghai, 1999, pp. 392-396 (in Chinese).

12) Chiu N. Y., Chang K. H., "The Illustrated Medicinal Plants of Taiwan,” Vol. 5, SMC Publishing Inc., Taipei, Taiwan, Republic of China, 1998, p. 194 (in Chinese).

13) Liu H. Y., "Flora of Taiwan," Vol. 4, ed. by Huang T. C., Department of Botany, National Taiwan University, Taipei, Taiwan, 1998, pp. $624-625$.

14) Nishioka I., Shoyakugaku Zasshi, 42, 1-11 (1988).

15) Tsay H. S., Gau T. G., Chen C. C., Plant Cell Rep., 8, 450-454 (1989).

16) Yamada Y., Shoyama Y., Nishioka I., Kohda H., Namera A., Okamoto T., Chem. Pharm. Bull., 39, 204-206 (1991).

17) Morimoto S., Goto Y., Shoyama Y., J. Nat. Prod., 57, 817-823 (1994).

18) Hiraoka N., Kodama T., Oyanagi M., Nakano S., Tomita Y., Yamada N., Iida O., Satake M., Plant Cell Rep., 5, 319-321 (1986).

19) Tsay H. S., Huang H. L., Plant Cell Rep., 17, 670-674 (1998).

20) Sagare A. P., Lee Y. L., Lin T. C., Chen C. C., Tsay H. S., Plant Sci., 160, 139-147 (2000)

21) Shrivastava N., Rajani M., Plant Cell Rep., 18, 919-923 (1999).

22) Paek K. Y., Yu K. J., Park S. I., Sung N. S., Park C. H., Acta Hort., 
390, 121-126 (1995).

23) Murashige T., Skoog F., Physiol. Plant., 15, 473-497 (1962).

24) Huang C. L., Hsieh M. T., Hsieh W. C., Sagare A. P., Tsay H. S., In Vitro Cell. Dev. Biol., 36, 220-224 (2000).

25) Chen C. C., Chen S. J., Sagare A. P., Tsay H. S., Bot. Bull. Acad. Sin., 42, 1-7 (2001).

26) Chueh F. S., Chen C. C., Sagare A. P., Tsay H. S., Planta Med., 67, 70-73 (2001).

27) Fernandez-Matellano L., Ollivier E., Diaz-Lanza A. M., Faure R., Balansard G., Planta Med., 61, 93 (1995).
28) Li Y. M., Jiang S. H., Gao W. Y., Zhu D. Y., Phytochemistry, 50, 101104 (1999).

29) Qian J., Hunkler D., Rimpler H., Phytochemistry, 31, 905-911 (1992).

30) Recio M. C., Giner R. M., Máñez S., Ríos J. L., Planta Med., 60, 232-234 (1994).

31) Baghdikian B., Lanhers M. C., Fleurentin J., Ollivier E., Maillard C., Balansard G., Mortier F., Planta Med., 63, 171-176 (1997).

32) Garg H. S., Bhandari S. P. S., Tripathi S. C., Patnaik G. K., Puri A., Saxena R., Saxena R. P., Phytotherapy Res., 8, 224-228 (1994). 